\title{
artigo
}

\section{Desafios da enfermagem frente ao parto humanizado: percepções de profissionais sobre a humanização em obstetrícia}

Challenges of nursing in the face of humanized childbirth: perceptions of professionals about humanization in obstetrics Retos de la enfermería frente al parto humanizado: percepciones de los profesionales sobre la humanización en obstetricia

\section{RESUMO}

Objetivo é sintetizar os conhecimentos científicos a respeito da humanização da assistência de enfermagem no parto natural, buscando destacar a importância da assistência humanizada durante o parto, apontando sobre seu papel no desenvolvimento de ações de humanização em um centro obstétrico de uma maternidade de referência. Método: A busca de dados foi realizada nas bases de dados: SciELO e Google Scholar a partir dos descritores em ciências da saúde: Enfermagem; Desafios e Parto humanizado, resultando na seleção final de 29 artigos. Resultados: A implantação de uma assistência de enfermagem humanizada durante o parto favorece o fortalecimento do vínculo profissional e paciente, garantindo o direito de escolha durante o trabalho de parto. Conclusão: Apesar das dificuldades com espaços inadequados e falta de matérias, a educação em saúde desempenhada pelos enfermeiros pode conscientizar a adesão ao parto natural como também a pratica da humanização por parte de outros profissionais de saúde.
\end{abstract}

DESCRITORES: Enfermagem; Desafios; Parto humanizado.

\section{ABSTRACT}

The objective is to synthesize scientific knowledge about the humanization of nursing care in natural childbirth, seeking to highlight the importance of humanized care during childbirth, pointing out its role in the development of humanization actions in an obstetric center of a reference maternity hospital. Method: The data search was performed in the following databases: SciELO and Google Scholar from the descriptors in health sciences: Nursing; Challenges and humanized delivery, resulting in the final selection of 29 articles. Results: The implementation of humanized nursing care during childbirth favors the strengthening of the professional and patient bond, ensuring the right to choose during labor. Conclusion: Despite the difficulties with inadequate spaces and lack of materials, health education performed by nurses can raise awareness of adherence to natural childbirth as well as the practice of humanization by other health professionals.

DESCRIPTORS: Nursing; Challenges; Humanized Childbirth.

\section{RESUMEN}

El objetivo es sintetizar conocimientos científicos sobre la humanización del cuidado de enfermería en el parto natural, buscando resaltar la importancia del cuidado humanizado durante el parto, señalando su rol en el desarrollo de acciones de humanización en un centro obstétrico de una maternidad de referencia. Método: La búsqueda de datos se realizó en las siguientes bases de datos: SciELO y Google Scholar a partir de los descriptores en ciencias de la salud: Enfermería; Retos y entrega humanizada, resultando en la selección final de 29 artículos. Resultados: La implementación de cuidados de enfermería humanizados durante el parto favorece el fortalecimiento del vínculo profesional y paciente, asegurando el derecho a elegir durante el parto. Conclusión: A pesar de las dificultades con los espacios inadecuados y la falta de materiales, la educación en salud realizada por enfermeras puede generar conciencia sobre la adherencia al parto natural así como la práctica de la humanización por parte de otros profesionales de la salud.

DESCRIPTORES: Enfermería; Desafíos; Nacimiento humanizado.

RECEBIDO EM: 06/05/2021 APROVADO EM: 18/06/2021 


\section{Mikael Henrique de Jesus Batista}

Enfermeiro pela Universidade Federal de Goiás. Mestre em Ensino em Ciências e Saúde pela Universidade Federal do Tocantins. Doutorando em Engenharia Biomédica pela Universidade Brasil. Docente do curso de Enfermagem. Faculdade de Colinas do Tocantins - Grupo UNIESP.

ORCID: 0000-0002-9277-8295

\section{Alanne Alves de Araujo}

Acadêmica de Enfermagem. Faculdade de Colinas do Tocantins - Grupo UNIESP.

ORCID: 0000-0003-2731-2947

\section{Ruan Feitosa dos Santos}

Enfermeiro. Especialista em Saúde Pública com ênfase em Estratégia Saúde da Família. Pós-graduando em Saúde Indígena. Fundação Municipal de Saúde de Pau D'arco, Tocantins.

ORCID: 0000-0002-8197-5760

\section{Daiara Vicentino Lima}

Acadêmica de Enfermagem. Faculdade de Colinas do Tocantins - Grupo UNIESP. Auxiliar administrativo. Prefeitura de Bandeirantes do Tocantins, Tocantins.

ORCID: 0000-0002-9493-1708

\section{Tainá Soares Nunes}

Enfermeira no Hospital Municipal de Colinas do Tocantins. Especialista em Urência e Emergência. Especialista em Terapia Intensiva Geral. Mestranda em Enfermagem pela UNESP.

Orcid: 0000-0002-0614-3841

\section{Ana Catarina de Moraes Souza}

Enfermeira. Docente de enfermagem da Faculdade de Colinas do Tocantins, Grupo UNIESP. Mestra em Promoção da Saúde pelo Centro Universitário Adventista de São Paulo-UNASP.

ORCID: 0000-0003-3549-0743

\section{INTRODUÇÃO}

$\mathbf{H}$ umanização atualmente é um termo amplamente discutido porque permeia os valores e práticas que envolvem as relações entre as pessoas. $\mathrm{O}$ atendimento humanizado deve estar centrado nas necessidades do cliente, não apenas em procedimentos e normas técnicas. Sendo que, essa pratica fazem uma grande diferença durante toda a assistência prestada à parturiente ${ }^{1}$.

O conceito de humanização do parto é diverso, onde alguns movimentos defendem esse conceito, com propósitos de respeitar a personalidade da mulher, usando-a como protagonista e buscando se adaptar ao processo de sua cultura, crenças, valores e diversidade a fim de promover um atendimento humanizado ${ }^{2}$.

De acordo com o Ministério da Saúde, as maternidades devem: Garantir que haja sala de parto. Mulheres grávidas tem o direito de seus acompanhantes estarem presentes; adota boas práticas realizar assistência ao parto de acordo com as recomendações da Organização Mundial de Saúde (OMS); fornece privacidade as mulheres que trabalham e dão à luz; reduza a taxa de cesárea, episiotomia e uso de ocitocina, trabalho de parto e parto humanizado; incentive a participação do pai no nascimento e promova o contato entre a mãe e o bebê após o parto ${ }^{3}$.

A assistência hospitalar ao parto deve ser segura e garantir os benefícios do progresso científico para todas as mulheres, mas fundamentalmente falando, deve permitir e estimular o exercício da cidadania feminina e salvar a autonomia da mulher no parto ${ }^{4}$.

Na ideia da humanização do parto, um dos grandes desafios que as enfermeiras obstétricas enfrentam em suas relações cotidianas é enfrentar e superar as barreiras à aplicação generalizada de estratégias não farmacológicas, mudando a cultura intervencionista do parto 5 .
De modo que é necessário que as instituições recomendem o desenvolvimento de métodos humanizados de parto, como a aplicação de estratégias não medicamentosas para aliviar a dor durante o parto, é óbvio que essas medidas não foram implementadas, são difíceis de introduzir e são difíceis de programar em vários centros obstétricos $^{6}$.

O processo de escolha do parto é muito importante, por isso, deve-se estar atento à saúde do recém-nascido e da mãe, antes de tudo, a gestante deve ser examinada sob orientação de profissionais capacitados e responsáveis. A importância dessa decisão será pautada, onde cada vez mais profissionais de enfermagem desempenham papel fundamental na assistência prestada ao parto, uma vez que estes se fazem presente durante todo o pré-natal ${ }^{7}$.

Ressalta-se que o profissional enfermeiro é muito importante no desenvolvimento do cuidado humanístico, pois proporciona à mulher que dá à luz a opor- 


\section{artigo}

Batista, M.H.J.; Araujo, A.A.; Santos, R.F.; Lima, D.V.; Nunes, T.S.; Souza, A.C.M.;

Desafios da enfermagem frente ao parto humanizado: percepções de profissionais sobre a humanização em obstetrícia

tunidade de experimentar diretamente durante o processo de parto por meio do uso de tecnologia e do atendimento psicológico e conforto 8 .

Os profissionais de enfermagem participaram da principal discussão sobre a saúde da mulher sobre o movimento social em defesa do PHPN. Nessa perspectiva, o Ministério da Saúde e outros órgãos governamentais consideram o enfermeiro como profissional plenamente capacitado, que pode cuidar da mulher nos diversos espaços reprodutivos de forma humanizada?

$\mathrm{O}$ processo de humanização do parto tem como foco o respeito às escolhas das mulheres e o direito aos direitos reprodutivos, dignidade, respeito e nenhuma violência de qualquer tipo ${ }^{10}$. Diante disso, é importante destacar para a sociedade que a humanização do parto é mais do que uma opção, neste sentido, o presente estudo traz em sua essência a seguinte pergunta norteadora: quais os desafios enfrentados pela Enfermagem na assistência ao parto humanizado? De modo que o objetivo é sintetizar os conhecimentos científicos a respeito da humanização da assistência de

enfermagem no parto natural, buscando destacar a importância da assistência humanizada durante o parto, apontando sobre seu papel no desenvolvimento de ações de humanização em um centro obstétrico de uma maternidade de referência.

\section{MÉTODOS}

Trata-se de uma revisão bibliográfica da literatura com de caráter quantitativo e exploratório, envolvendo artigos científcos publicados em português nas bases de dados científicos: Scientific Electronic Library Online (SciELO) e Google Scholar, publicados entre os anos de 2015 e 2021 através da utilização do cruzamento do descritores em Ciências da Saúde: Enfermagem; Desafios e Parto humanizado, utilizando o boleando AND como conectivo deste, processo sistemático utilizada em ambas plataformas supracitadas.

Os artigos foram pesquisados entre janeiro e abril de 2021. Nesse período, também foram pesquisados, lidos, selecionados e analisados os artigos que faram parte dos resultados e discussões deste estudo.

\section{Tabela 1- Dados do levantamento bibliográfico segundo as bases de dados} supracitadas.

\section{DESAFIOS DA ENFERMAGEM FRENTE AO PARTO HUMANIZADO: PERCEPÇÕES DE} PROFISSIONAIS SOBRE A HUMANIZAÇÃO EM OBSTETRICIA

\section{LEVANTAMENTO BIBLIOGRÁFICO}

Scientific Eletronic Library Online (SciELO)

Google Scholar

Total

\section{RESULTADOS OBTIDOS}

ARTIGO
SELECIONADO

1.235 Artigos
1.834 Artigos

11 Artigos

18 Artigos

29 Artigos
ARTIGO EXCLUÍDO 1.224 Artigos 1.816 Artigos 3040 Artigos
Descritores: Enfermagem; Desafios; Parto Humanizado. Fonte: Pesquisa intitulada: Desafios da enfermagem frente ao parto humanizado: percepções de profissionais sobre a humanização em obstetrícia.
Os critérios de inclusão dos estudos incluíram: a) Artigos completos disponível na íntegra; b) Ter a versão na língua portuguesa; c) Ser artigos gratuitos. Houve a exclusão de artigos duplicados; os que após leitura dos resumos se enquadraram com fuga ao tema proposto e os que não se enquadraram nos critérios de inclusão supracitados.

Após a seleção e aplicação dos critérios de inclusão e exclusão, a amostra final é de 29 artigos, sendo 11 da plataforma de pesquisa SciELO e 18 do Google Scholar.

Posteriormente, realizou-se a análise crítica-reflexiva dos artigos e houve a categorização dos assuntos, culminando nas categorias: Aspectos intrínsecos à humanização no trabalho de parto normal; Importância da assistência humanizada durante o parto; A enfermagem no contexto da assistência humanizada ao parto.

\section{RESULTADOS}

Os artigos incluídos para esta revisão são estudos que versão sobre os desafios inerentes ao parto humanizado atualmente, após análise crítica-reflexiva dos estudos selecionados, desenvolveu-se a tabela abaixo sumarizando os artigos que serão discutidos adiante com essa temática, sendo está o eixo norteador da discussão.

\section{DISCUSSÃO}

A assistência de enfermagem no parto humanizado promove a integração na presença de pares e participação da mulher no processo de escolha de um melhor tipo de parto, onde os profissionais de enferma-

Tabela 2. Descrição dos artigos que fazem parte da amostragem final deste estudo.

$$
\begin{array}{lll}
\text { TÍTULO ANO OBJETIVO } &
\end{array}
$$

o objetivo de conhecer, através da literatura

Desafios da enfermagem frente ao parto: uma revisão integrativa. científica, como a assistência de enfermagem tem contribuído na humanização do

2019 parto, buscando: Entender como é feito o acolhimento da gestante, os métodos não farmacológicos utilizados, o respeito a individualidade e privacidade da parturiente.

\section{RESULTADO}

Após a revisão integrativa, seguindo os critérios de inclusão e exclusão da amostra, foi possível selecionar os sete artigos que se integraram à proposta dessa revisão, onde buscou compreender a contribuição da enfermagem para a humanização do parto, através da análise do seu papel na promoção da autonomia, protagonismo, individualidade e privacidade da gestante além das práticas de acolhimento e técnicas não farmacológicas para alívio da dor. 
Estratégias não farmacológicas do enfermeiro obstetra frente à dor no trabaIho de parto: produção científica entre 2009 e 2014

A violência obstétrica no cotidiano assistencial e suas características.

Qualidade da Assistência Pré-natal no sistema Único de Saúde.

Limites da assistência ao parto no centro de parto normal de uma maternidade pública

Uma Revisão Bibliográfica. Especialização em Saúde Pública

Análise de partos acompanhados por enfermeiras obstétricas na perspectiva da humanização do parto e nascimento

Parto Humanizado: Um direito a ser respeitado
Identificar quais as estratégias não farmacológicas frente à dor da parturiente mais ressaltadas pelos artigos, descrever os recursos e métodos utilizados pelo

Enfermeiro obstetra para implementar a humanização do trabalho de parto.

analisar a produção científica sobre a violência obstétrica identificando e dis-

2018 cutindo suas principais características no cotidiano da assistência ao ciclo gravídico e puerperal.

2018

Avaliar a qualidade da assistência pré-natal a partir do Programa de Humanização no Pré-Natal e Nascimento.

2018

O objetivo de compreender e descrever as crenças e os valores que norteiam as práticas dos profissionais em um cpn.

Realizar uma revisão narrativa de literatura sobre a humanização no serviço público de saúde, para posterior aplicação prática dos conhecimentos levantados às equipes de saúde da Secretaria Municipal de Saúde de Curitiba/PR.

Analisar partos acompanhados pelas 2016 enfermeiras obstétricas relacionando sua prática com a política de humanização do parto e nascimento.

Descrever a assistência de enfermagem prestada no parto normal humanizado
O Ministério da Saúde (BRASIL, 2001a, p. 31) considera que a dor do parto deve ser aliviada e relata que algumas medidas, isoladas ou combinadas, são eficazes na redução da sensação dolorosa, destacando como medidas não farmacológicas as técnicas de relaxamento, os exercícios respiratórios, a deambulação e a mudança de posição que não seja a deitada durante o parto. Ressalta que a mudança de posição é excelente para a dilatação do colo ao aumentarem a intensidade e a eficiência da contração interina.

as publicações concentram-se a partir de 2015 com desenhos metodológicos de natureza quantitativa e qualitativa. Na discussão, primeiramente, aborda-se o conceito de violência obstétrica e suas diferentes formas de ocorrência na assistência. Em sequência, são apresentadas as interfaces do fenômeno com reflexões relacionadas à concepção de gênero, aos diferentes atores envolvidos, à institucionalização, à invisibilidade e à banalização do evento. Por fim, são apresentadas as estratégias de enfrentamento perpassando pela formação acadêmica, pela conscientização das mulheres, pelas propostas de mobilização social, pela construção de políticas públicas e leis.

Pouco mais da metade das puérperas haviam iniciado seu acompanhamento pré-natal antes da $16^{\mathrm{a}}$ semana gestacional e também realizaram seis ou mais consultas $(66,9 \% ; n=174$, cada variável). Somente $41,5 \%(n=108)$ das participantes receberam informações sobre a maternidade de referência para o parto e $42,7 \%(n=111)$ sobre as atividades para facilitar o parto. Quanto aos exames, foi identificada elevada cobertura no primeiro trimestre, com discreta redução no terceiro.

as limitações para o desenvolvimento da assistência humanizada ao parto estavam relacionadas, não somente aos problemas relativos à precariedade da estrutura física e de recursos humanos ou aos déficits provocados pelo financiamento insuficiente da assistência ao parto.

A principal mudança do serviço para a prática da humanização está nas pessoas: no usuário disposto a mudar hábitos e aprender novas formas de ter saúde; no trabalhador sentir-se motivado a ser aproximar mais do usuário, estabelecendo elos de comunicação e vínculo e, no gestor, incentivando e oportunizando essa prática.

As primigestas representaram $44,16 \%$ das parturientes. As intervenções mais recorrentes foram a administração de ocitocina e a amniotomia. A posição horizontal dorsal ocorreu em $12,89 \%$ dos partos. A incidência de episiotomia foi de $15,52 \%$.

Mostra que apesar das dificuldades encontradas diariamente, a atuação dos enfermeiros vem, pouco a pouco conquistando seu espaço na obstetrícia, estabelecendo através de sua evolução, vínculos que promovem uma assistência qualificada e bem fundamentada na humanização 


\section{artigo}

Batista, M.H.J.; Araujo, A.A.; Santos, R.F.; Lima, D.V.; Nunes, T.S.; Souza, A.C.M.

Desafios da enfermagem frente ao parto humanizado: percepções de profissionais sobre a humanização em obstetrícia

A humanização do nascimento: percepção dos profissionais de saúde que atuam na atenção ao parto.

humanização da assistência de enfermagem no trabalho de parto natural

o trabalho de enfermeiras(os) obstetras em goiânia: identidade, políticas de saúde e desafios profissionais

O profissional enfermeiro como educador: um olhar para atenção primária à saúde e o NASF Revista interdisciplinar.

O parto humanizado e o seu impacto na assistência à saúde.

Humanização do Parto: Atuação dos Enfermeiros

Significado de parto humanizado para gestantes

Compreender o significado de parto humanizado na concepção de gestantes.

O presente estudo objetivou conhecer as percepções, as expectativas e os co-

Percepções, expectativas e conhecimentos sobre o parto normal: relatos de experiência de parturientes e dos profissionais de saúde.

objetivo foi conhecer a percepção dos profissionais de saúde que atuam na assistência ao parto sobre a humanização do processo de nascimento.

Objetiva-se sintetizar os conhecimentos científicos a respeito da humanização da assistência de enfermagem no parto natural

O objetivo do texto é destacar como a categoria tem atuado na cidade de Goiânia, seus principais desafios e dificuldades no cotidiano laboral. De modo a contextualizar as declarações em quadro abreviado serão apresentados os principais marcos legais e as principais políticas de saúde voltados à categoria.

Objetivou-se analisar a importância da equipe de matriciamento e do enfermeiro como membro multiplicador de saúde e como educador. Este artigo consiste em uma revisão bibliográfica prospectiva de natureza qualitativa.

Fazer uma análise sobre o parto humanizado e suas vicissitudes no que tange aspectos Fisiológicos psicossociais, tanto quanto especificidades tecnocráticas na sua operacionalização.

objetivo proporcionar maior familiaridade com o problema com vista a torná-lo mais explícito ou construir hipóteses. Pode-se dizer que tais pesquisas têm como finalidade principal o aprimoramento de ideias ou a descoberta de intuições.

2012 nhecimentos de puérperas em relação à experiência do parto normal, assim como os procedimentos utilizados pelos profissionais da saúde para a humanização do parto.
Os resultados mostram que a humanização na assistência ao nascimento ainda não é uma prática presente na maioria dos hospitais estudados e que os profissionais não estão preparados para prestar um atendimento humanizado e com qualidade tanto para a mãe quanto para o recém-nascido.

Além disso, destaca-se também a deficiência do pessoal de enfermagem e a grande demanda de trabalho, pois distanciam as enfermeiras do cuidado, o que acaba revertendo em prejuízo para a assistência prestada à parturiente, recém-nascido e familia, tornando as práticas mais verticais e tecnicistas.

A Pesquisa em andamento desde 2013 consiste no consequente aprofundamento da pesquisa realizada de agosto de 2011 a julho de 2012, durante o último ano de graduação, em que se pretendeu perceber o cotidiano de trabalho de técnicas de enfermagem de uma maternidade pública da cidade que preconiza o parto humanizado.

Após análise detalhada dos artigos, verificou-se que apenas 20 abordavam realmente o tema em questão, sendo, portanto, utilizados na construção desse estudo. Os artigos foram selecionados e lidos minuciosamente para compor essa pesquisa.

Dentre o averiguado no acervo bibliográfico, destaca-se os principais benefícios encontrados como a importância do acompanhante, abandono de procedimentos desnecessários, protagonismo da mulher e maior estimulo à amamentação.

Observou-se a ascensão da enfermagem obstétrica através de incentivos de políticas públicas e estas consolidam o processo de humanizar.

o tema central desvelado foi: compreendendo o parto humanizado como aquele em que o profissional tenha como prerrogativa o relacionamento empático e a competência técnica que propicie à mulher a vivência de um trabalho de parto e parto fisiológico como protagonista durante este processo.

A experiência da parturição foi percebida pela maioria das mulheres como extremamente dolorosa e sofrida, compensada, no entanto, pela atenção, apoio e carinho recebidos de alguns profissionais e acompanhantes, que contribuíram para uma visão satisfatória do parto normal. Entre os profissionais evidenciou-se dificuldade em conceituar sobre a temática da humanização do parto. 
gem devem estar capacitados para prestar uma assistência de qualidade. Deste modo, com base nos achados neste estudo, a partir da análise desenvolvida, discute-se as categorias supracitadas logo abaixo ${ }^{7}$.

\section{Aspectos intrínsecos à humanização no trabalho de parto normal}

A humanização deve compreender pelo menos dois fatores para ser implementada: primeiro, a secretaria de saúde tem a responsabilidade de prestar atenção adequada e digna à mulher, sua família e ao recém-nascido, portanto, a organização dos profissionais de saúde e das secretarias de saúde é extremamente necessária 5 .

Neste sentido, ${ }^{11}$ evidencia que no Brasil, a assistência ao parto e nascimento é prestada há décadas, e a mesma, é marcada por importantes mudanças ocasionadas pelo processo de institucionalização, levando a um aumento da condição médica do corpo feminino, promovendo sua desfragmentação, e muitas das vezes morbidade pelo uso indevido de intervenções desnecessárias para mulheres e bebês nesse processo.

Porém, por se tratar de um processo consolidado no modelo biomédico em assistência à saúde, há alguns obstáculos na implementação do atendimento humanizado para a parturiente, nos quais envolvem a compreensão inadequada das mulheres, de suas famílias e de seus companheiros sobre os direitos reprodutivos no parto e saúde reprodutiva, e a falta de orientação e apoio para as atividades de representação das mulheres e suas famílias ${ }^{12}$.

Visto que a humanização tem como objetivo colocar a gestante no papel principal, verificam-se barreiras neste processo como, por exemplo, a presença de uma hierarquia entre os medidores de saúde e a parturiente, atualmente na sociedade os profissionais são vistos como principais no parto, pois, possuem o saber e as técnicas, fazendo com que as gestantes não tenham participação no processo que o seu corpo está passando ${ }^{13}$.

A humanização do parto e nascimento exige dos atores envolvidos neste processo, esforços para que as condutas e os procedimentos desnecessários e intervencionistas sejam minimizados, tanto para a mulher quanto para o recém-nascido?.

$O$ processo de humanização no atendimento é uma forma de identificar e valorizar o sentido que o sofrimento pelo adoecer traz ao paciente, reconhecendo e trabalhando as relações interpessoais neste processo ${ }^{7}$.

$\mathrm{O}$ pesquisador Reis ${ }^{14}$ enfatiza a extrema importância da formação e atuação da equipe de enfermagem, cabendo aos especialistas orientar a mãe e os familiares sobre a evolução do parto, compreender como controlar e planejar estratégias para superar as dificuldades e minimizar o trauma e a dor de ambas as partes.

A escolha de parto e algo muito difícil a ser decidido para a mulher, e também para a sua família sendo que esta escolha é uma dúvida repetida em meio às mulheres, a escolha deve sempre prevalecer o bem estar da parturiente, tendo em vista a minimização dos riscos tanto a saúde da mãe como a do recém-nascido. No qual durante o estudo abordara a escolhas das mulheres pelo tipo de parto e suas consequências 5 .

A humanização proposta pelo parto humanizado entende a gestação e o parto como eventos fisiológicos perfeitos, cabendo a obstetrícia acompanhar o processo, buscando aperfeiçoá-lo, sempre respeitando a individualidade da gestante nos mais diversos aspectos ${ }^{15}$.

\section{Importância da assistência humani- zada durante o parto}

O desenvolvimento das ações de enfermagem pode proporcionar à parturiente maior segurança e conforto durante o parto, mantendo sempre uma escuta ativa e atenta8. Deste modo, ${ }^{1}$ aponta-se a humanização como imprescindível em todas as etapas do processo de gestação e parto, cuja assistência nessa etapa deve respeitar as restrições, desejos e medos da parturiente.

Neste sentido, ${ }^{16}$ refere-se que a relação enfermeiro-paciente precisa ser dinâmica para integrar o conhecimento do paciente ao conhecimento científico e manter sua autonomia, acolhendo e proporcionando segurança a estas. De modo que, ao se referir ao binômio enfermeiro-paciente, ressalta-se que a assistência prestada no parto e puerpé- rio está condicionada a mudança de atitudes de todos os envolvidos neste processo o caminho para garantir o alcance de qualidade consiste em trabalhar a humanização do atendimento as parturientes e familiares ${ }^{17}$.

No momento do parto existem muitas dificuldades para prestar uma assistência humanizada às mulheres e algumas delas são: a necessidade de profissionais capacitados para tal; disponibilidade de recursos tecnológicos e infraestrutura adequada da instituição; além das condições fisiológicas e psicológicas da mulher no momento do parto que necessita de atenção e apoio emocional ${ }^{18}$.

O contato entre profissional e cliente ainda é uma relação assimétrica e as mulheres por se sentirem menos capacitada para fazer escolhas e fazer valer seus desejos não participam de decisões técnicas estabelecidas pelos profissionais da saúde, isto é evidenciado pela prática cada vez maior de intervenções cirúrgicas desnecessárias, o que desvaloriza o parto natural. Assim sendo, existe a necessidade da institucionalização do parto humanizado pelo Ministério da saúde em toda rede hospitalar 15.

Pois, com o apoio do fortalecimento por parte do Ministério da Saúde é possível realizar uma assistência humanizada ao parto e reduzir a prática de métodos invasivos, de modo que a formação continuada dos profissionais envolvidos na maternidade da instituição a respeito do parto humanizado se torna essencial ${ }^{19}$.

\section{A enfermagem no contexto da assis- tência humanizada ao parto}

O significado de assistência humanizada ao parto aborda diversos aspectos, alguns deles estão relacionados a mudanças na cultura hospitalar, e as organizações de ajuda são de fato planejadas para atender às necessidades das mulheres e de suas famílias, de modo que é necessário transformar o espaço hospitalar em um ambiente mais humano e mais favorável para programar a prática de enfermagem humanizada ${ }^{20,13}$.

Nos últimos anos, diversos estudiosos e organizações não governamentais têm demonstrado suas preocupações com a medicalização excessiva do parto, propondo modificações no modelo de assistência ao parto, 


\section{artigo}

principalmente naqueles de baixo risco ${ }^{21}$.

Nesse modelo humanizado, o trabalho da enfermagem requer profissionais sem preconceitos, prestando serviços sem prejuízos e minimizando intervenções, sendo o respeito, solidariedade, apoio, orientação e incentivo fatores que influenciam diretamente a assistência humanitária? .

${ }^{18} \mathrm{~A}$ atuação do enfermeiro é fundamental na humanização do parto, pois contribui com a gestante explicando o desenvolvimento do parto, esclarecendo suas dúvidas e evitando complicações. As orientações fornecidas, o acolhimento da gestante, a substituição de procedimentos, às vezes desnecessários, por condutas menos invasivas e não farmacológicas que minimizem a dor e o sofrimento são estratégias apontadas para o fortalecimento do modelo humanizado. Nessa perspectiva o movimento pela humanização do parto perpassa pela mudança do paradigma de gravidez ser vista como doença, para que se possa gozar de ações humanizadas ${ }^{22,23}$.

\section{CONCLUSÃO}

Neste estudo, torna-se evidente a importância dos profissionais de enfermagem durante o parto e pós-parto, em especial em fornecerem cuidados humanizados com escuta ativa e atenta, o que é capaz de proporcionar maior segurança e conforto, demonstrando também que o estabelecimento de vínculo com o paciente é fundamental para a compreensão suas necessidades e ofertar uma assistência de qualidade.

\section{REFERÊNCIAS}

1. Porto, AAS, Costa LP, Velloso, NA. Humanização da assistência ao parto natural: uma revisão integrativa. Rev. Ciência e Tecnologia. v.1, n.1, p 12-19. Rio Grande do Sul. 2015.

2. Dodou HD, Rodrigues DP, Oriá MOB. O cuidado à mulher no contexto da maternidade: caminhos e desafios para a humanização. Rev Fund Care Online. 2017 jan/mar; 9(1):222-230.

3. Cordeiro EL, et al. A humanização na assistência ao parto e ao nascimento. Rev. Enferm. UFPE, Recife, v. 12, n. 8, p. 2154-62, ago., 2018.

4. Santos IS, Okazaki EIFJ. Assistência de Enfermagem ao parto humanizado. Rev. Enferm. UNISA.v.1, n.13, p.64 - 8, 2017.

5. Pinto LC, et al. Estratégias não farmacológicas do enfermeiro obstetra frente à dor no trabalho de parto: produção científica entre 2009 e 2014. Revista Ciência Atual. 2018. Rio de Janeiro, v. $11 n^{\circ}$ 2. p. 2-21.

6. Ribeiro JL, et al. Os desafios da humanização na assistência de enfermagem ao parto normal em um hospital municipal do Estado do Tocantins. 2019. Revista Multidebates, v.3, n.1. p. 273-286.

7. Silva MFGD. 0 trabalho de enfermeiras/os obstetras na assistência ao parto e nascimento em Goiânia: Políticas de humanização, desafios de mercado e relações de trabalho. Dissertação (mestrado). Universidade Federal de Goiás. Programa de Pós-Graduação em Sociologia. 2015.

8. Souza DB. et al. Boas práticas de atenção ao parto e ao nascimento e os desafios para a humanização do cuidado. 2018. Seminário de Iniciação Cientifica. Universidade do Estado de Santa Catarina. $28^{\circ}$ SIC UDESC.

9. Santos Júnior PS. et al. Desafios da enfermagem frente ao parto: uma revisão integrativa. Rev. Multidisciplinar em Saúde. 2019. Trabalho de Conclusão de Curso (graduação). Universidade Tiradentes. Departamento de enfermagem. Aracajú.

10. Nagahama EEI, Santiago SM. Práticas de atenção ao parto e os desafios para humanização do cuidado em dois hospitais vinculados ao Sistema Único de Saúde em município da Região Sul do Brasil. Cad. Saúde Pública, v. 24, n. 8, p. 1859-1868, 2018.

11. Jardim DMB, Modena CM. A violência obstétrica no cotidiano assistencial e suas características. Rev. Latino-Am. Enfermagem, n. 26, p. 1-12, 2018.
12. Moura IKM, et al. O profissional enfermeiro como educador: um olhar para atenção primária à saúde e o NASF Revista interdisciplinar. 2015. Jan-mar. v 8. № 1.

13. Barros LP, et al., 0 parto humanizado e o seu impacto na assistência à saúde. RESU [Internet]. [s.n.], 2015. 67 p. v. 3.

14. Reis CSC. et al. Análise de partos acompanhados por enfermeiras obstétricas na perspectiva da humanização do parto e nascimento. Rev. Fund. Care Online, v. 8, n. 4, p. 4972-4979, out.-dez 2016.

15. Dantas DS, et al. Qualidade da Assistência Pré-natal no sistema Único de Saúde. Rev enferm UFPE on line., Recife, 12(5):1365-71, maio., 2018.

16. Matei EM, et al. Parto Humanizado: Um direito a ser respeitado. Centro Universitário S. Camilo, São Paulo, v.9, n.2, p.16 - 26, abr - jun 2015.

17. Brasil. Ministério da Saúde. Secretaria Executiva. Programa Humanização do Parto: humanização no pré-natal e nascimento. Reimpressão. Brasília (DF): Ministério da Saúde; 2016.

18. Silvani CMB. Parto humanizado - Uma Revisão Bibliográfica. Especialização em Saúde Pública. Porto Alegre: Universidade Federal do Rio Grande do Sul; 2017.

19. Souza TG, Gaíva MAM, Modes PSSA. A humanização do nascimento: percepção dos profissionais de saúde que atuam na atenção ao parto. Rev Gaúcha Enferm., v. 32, n. 3, 479-486, 2016 20. Nunes MCM. Entre o idealizado e o possível: limites da assistência ao parto no centro de parto normal de uma maternidade pública [dissertação]. São Paulo (SP): Programa de Pós-graduação em Enfermagem, Universidade de São Paulo; 2018.

21. Almeida OSC, Gama ER, Bahiana PM. Humanização do Parto: Atuação dos Enfermeiros. Revista Enfermagem Contemporânea. 2015 Jan/Jun.; 4(1):79-90

22. Versian CC, et al. Significado de parto humanizado para gestantes. Rev. Pesqui. (Univ. Fed. Estado Rio J., Online); 7(1): 19271935, jan.-mar. 2015.

23. Pinheiro BC, Bittar CML. Percepções, expectativas e conhecimentos sobre o parto normal: relatos de experiência de parturientes e dos profissionais de saúde. Aletheia [Internet]. 2012. 